\title{
Highly Transparent Nanocomposites Based on Poly(vinyl alcohol) and Sulfated UV-Absorbing Wood Nanofibers
}

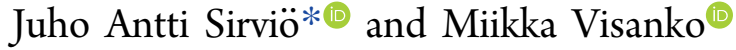 \\ Fibre and Particle Engineering Research Unit, University of Oulu, P.O. Box 4300, 90014 Oulu, Finland
}

Supporting Information

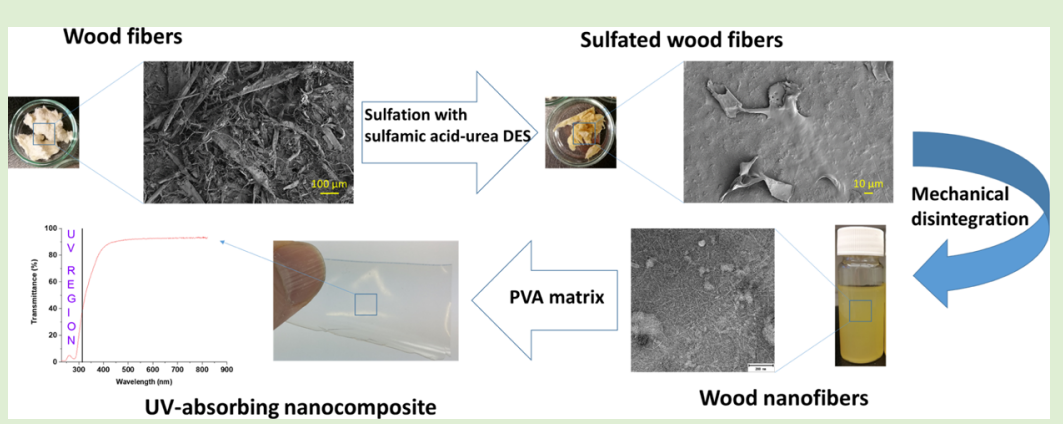

ABSTRACT: Unbleached lignocellulose fibers were studied for the fabrication of wood-based UV-absorbing nanofibers and were used to produce transparent nanocomposites. Groundwood pulp (GWP) and sawdust were selected as raw materials thanks to their low processing degree of fibers and abundant availability as a low-value industrial side stream. Both materials were first sulfated using a reactive deep eutectic solvent. The sulfated wood and sawdust nanofibers (SWNFs and SSDNFs, respectively) were fabricated using a mild mechanical disintegration approach. As a reference material, sulfated cellulose nanofibers (SCNFs) were obtained from bleached cellulose pulp. Our results showed that both GWP and sawdust exhibited similar reactivity compared with bleached cellulose pulp, whereas the yields of sulfated lignin-containing pulps were notably higher. The diameters of both SWNFs and SSDNFs were approximately $3 \mathrm{~nm}$, which was similar to those of the SCNFs. When 10 wt \% of lignin-containing nanofibers were mixed together with poly(vinyl alcohol), the fabrication of nanocomposites with only a minimal decrease in transparency in the visible light spectrum was achieved. Transmission in the UV region, on the other hand, was significantly reduced by SWNFs and SSDNFs, whereas SCNFs had only a minor UV-absorbing property. Although the reinforcing effect of lignin-containing nanofibers was lower compared with that of SCNFs, it was comparable with those of other UV-absorbing additives reported in the literature. Overall, the wood-based UV-absorbing nanofibers could have a valuable use in optical applications such as lenses and optoelectronics.

\section{INTRODUCTION}

Biomass-based nanomaterials have gained a significant amount of scientific and industrial attention for their large variety of applications. These nanomaterials from bioresources include those produced from natural polymers such as cellulose, ${ }^{1}$ chitosan, ${ }^{2}$ lignin, ${ }^{3}$ and starch. ${ }^{4}$ Compared with inorganic nanomaterials (e.g., metal nanoparticles), nanosized natural polymers are highly abundant, renewable, generally low in toxicity, and often biodegradable and biocompatible. As such, biomass-based nanomaterials have been studied for various applications, including tissue scaffolds, ${ }^{5,6}$ barrier materials, ${ }^{7,8}$ reinforcements for composite materials, ${ }^{9-11}$ and substrates for optoelectronics. $^{12,13}$

Lignocellulose is one of the most potential feedstocks for biobased nanomaterials thanks to its main components consisting of cellulose, lignin, and hemicelluloses. Nanocelluloses-including cellulose nanofibers $(\mathrm{CNFs})^{14}$ and cellulose nanocrystals (CNCs) ${ }^{15}$ together with other minority types of nanosized cellulose (e.g., spherical and fiber-like regenerated cellulose nanoparticles ${ }^{16,17}$ ) - have been inten- sively studied during recent years and have been utilized in a vast variety of applications. In general, nanocelluloses are produced from bleached cellulose fibers from which the lignin and hemicelluloses are in most cases, removed to a high extent.

Recently, there has been an increased interest to produce nanomaterials directly from lignocellulose, which contains all or most of the noncellulosic components, as it has several advantages. ${ }^{18-23}$ The use of unbleached lignocellulose fibers could significantly improve the environmental feasibility of biomass-based nanomaterials, thanks to exclusion of hazardous bleaching chemicals. In addition, the use of energy could be reduced, whereas the yield of the pure lignocellulose is higher due to the preservation of its original components. Additionally, the use of low-processed lignocellulose can improve the economic feasibility of lignocellulose nanomaterials because of the notably lower price of unbleached pulp.

Received: $\quad$ March 28, 2019

Revised: April 26, 2019

Published: April 29, 2019 
The preservation of lignocellulosic components, such as lignin, could provide additional functional properties to the elaborated materials. For example, we recently demonstrated that the preparation time of nanopapers decreased when using wood nanofibers (WNFs) with a high-lignin content and that the intact lignin aided the redispersion of dried nanofibers. ${ }^{20}$ The preservation of lignin in nanofibers can also improve the hydrophobicity of otherwise highly hydrophilic nanopapers; moreover, it enhances the thermal stability of nanopapers. ${ }^{24}$ Lignin is also known to form complexes with various metals, and as part of a lignocellulosic nanomaterial, it could be used for the removal of toxic heavy metals from natural and waste water. $^{25,26}$ Furthermore, thanks to the presence of a large number of phenolic groups, lignin has some antioxidant properties as well. ${ }^{27,28}$

Lignin is a highly conjugated aromatic component, making it an efficient UV absorbent. ${ }^{29,30} \mathrm{UV}$-absorbing properties are desired in many applications, such as contact lenses ${ }^{31}$ and in optoelectronics (e.g., solar cells ${ }^{32}$ and organic light-emitting diodes $^{33}$ ). By introducing biobased UV absorbents to electronic devices, malfunctions caused by overexposure of UV radiation could be reduced. UV-absorbing contact lenses and optoelectronics require a minimal absorbance or reflectance of visible light - that is, they must have high visible light transparency. Therefore, nanomaterials with dimensions smaller than the wavelength of visible light are one of the most potential UV-protecting materials for such components.

In this study, the production of nanofibers containing lignin (i.e., WNFs) was studied. WNFs were produced from groundwood pulp (GWP). Additionally, the possibility for direct utilization of an industrial side stream of sawmills was studied by using sawdust as an alternative raw material. Prior to mechanical disintegration with the microfluidizer, GWP and sawdust were sulfated using the reactive deep eutectic solvent (DES). The feasibility to use WNFs in material applications was studied by fabricating nanocomposites, which were produced by mixing poly(vinyl alcohol) (PVA) with $10 \%$ of wood-based nanofibers. The properties of WNF as a UVabsorbing additive and reinforcement agent were compared with sulfated cellulose nanofibers (SCNFs) obtained from bleached cellulose pulp.

\section{MATERIALS AND METHODS}

Materials. Unbleached spruce GWP and sawdust were obtained as a never-dried form, whereas the softwood-dissolving cellulose pulp was obtained as dry sheets. ${ }^{34}$ For the GWP and sawdust, they were first oven-dried $\left(24 \mathrm{~h}\right.$ at $\left.60{ }^{\circ} \mathrm{C}\right)$ before use, and for the dissolving pulp, it was first disintegrated in water and then filtered, washed with ethanol, and dried at $60^{\circ} \mathrm{C}$ for $24 \mathrm{~h}$ in oven. Standard methods were used to analyze the compositions of the raw materials: the lignin content with TAPPI T 222 om-02 standard, the acetone-soluble extractives with TAPPI T 280 standard pm-99, and the hemicellulose and degraded cellulose portions with TAPPI T 212 om- 02 standard. The results of the analysis are presented in Table 1 . Before the use, the sawdust was ground with an Ultra-Centrifugal Mill ZM 200 (Retsch, Germany) by using a sieve size of $250 \mu \mathrm{m}$.

Urea (Borealis Biuron, Austria), sulfamic acid (Sigma-Aldrich, Germany), and PVA (Mowiol 3-96, 96.8-97.6 mol \% hydrolysis, Sigma-Aldrich, Germany) were used as received. For all steps requiring water, unless stated otherwise, deionized water was used.

Sulfation of Cellulose. Sulfation of wood and cellulose samples was performed in a similar manner as previously presented using DESs based on sulfamic acid and urea as a reagent and reactions media. ${ }^{35}$ Briefly, the components of DES were mixed together using a magnetic stirrer in an oil bath at $80{ }^{\circ} \mathrm{C}$ with a molar ratio (sulfamic
Table 1. Lignin, Hemicellulose, and Acetone-Soluble Content of the Raw Materials

\begin{tabular}{lccc} 
& lignin & hemicellulose & acetone soluble \\
GWP & 27.4 & 13 & 2.2 \\
sawdust & 28.6 & 19.7 & 1.2 \\
dissolving pulp & $<0.5$ & 3.5 & 0.17 \\
\hline
\end{tabular}

acid/urea) of 1:2 until a clear solution was obtained. Then, GWP, ground sawdust, and dissolving pulp were each separately added to the DES, Next, the reaction temperature was increased to $150^{\circ} \mathrm{C}$, and the reaction was allowed to proceed for $30 \mathrm{~min}$. The product was then filtrated and washed on the filter paper with water using several washing cycles until $\mathrm{pH}$ of the filtrate was neutral. Finally, the product was collected and stored at $4{ }^{\circ} \mathrm{C}$.

Elemental Analysis of Sulfated Cellulose. The sulfated wood and cellulose samples were dried in an oven at $60{ }^{\circ} \mathrm{C}$ overnight. The nitrogen and sulfur contents of the samples were analyzed using the PerkinElmer CHNS/O 2400 Series II elemental and LECO CS-200 carbon-sulfur analyzers, respectively. The degree of substitution (DS) was calculated by using eq $1 .^{36}$

$$
\text { DS }=\frac{S \times 162.15}{3206-(S \times 97.10)}
$$

In eq $1, S$ represents the sulfur content, 162.15 is the molecular weight $(\mathrm{mmol} / \mathrm{g})$ of the anhydroglucose unit, and 97.10 is the molecular weight $(\mathrm{mmol} / \mathrm{g})$ of the ammonium sulfate group.

Disintegration of Sulfated Cellulose into Nanofibers. The never-dried sulfated wood fibers were diluted to a consistency of $1 \mathrm{wt}$ $\%$ ( $0.5 \mathrm{wt} \%$ for the sulfated dissolving pulp) in water, mixed for 30 min using a magnetic stirrer at room temperature, and then passed twice at a pressure of 1000 bar through the 400 and $200 \mu \mathrm{m}$ chambers of a microfluidizer (Microfluidics M-110EH-30, USA) to disintegrate the cellulose GWP, ground sawdust, and dissolving pulp to sulfated WNFs (SWNFs), sulfated sawdust nanofibers (SSDNFs), and SCNFs, respectively.

Diffuse Reflectance Infrared Fourier Transform Spectroscopy. The chemical characterization of pristine and sulfated cellulose was performed using diffuse reflectance infrared Fourier transform spectroscopy (DRIFT). The spectra were collected with a Bruker Vertex $80 \mathrm{v}$ spectrometer (USA) from freeze-dried samples. The spectra were obtained in the $600-4000 \mathrm{~cm}^{-1}$ range, and 40 scans were taken at a resolution of $2 \mathrm{~cm}^{-1}$ from each sample. Spectra were normalized to the band at $1001 \mathrm{~cm}^{-1} \cdot 37$

Transmission Electron Microscopy. The morphological features of the fabricated SCNF were analyzed with a JEM-2200FS transmission electron microscope (JEOL, Japan). Each sample was prepared by diluting it in ultrapure water (around $0.05 \%$ ). The carbon-coated copper grid was prepared by coating it with polylysine. Here, a small droplet of polylysine ( $0.1 \%$ solution in water) was placed on top of the grid and allowed to adhere for $3 \mathrm{~min}$. The excess polylysine was removed from the grid by touching the droplet with the corner of a filter paper. A small droplet of the diluted SCNF sample was then placed on top of the grid. Sample excess was similarly removed with a filter paper as well. Next, the samples were negatively stained by placing a droplet of uranyl acetate ( $2 \%$ in water) on top of each specimen. Excess of uranyl acetate was removed as described above. Finally, the grids were dried at room temperature and analyzed at $200 \mathrm{kV}$ under standard conditions. The dimensions of nanofibers were measured by determining about 500 individual nanofibers with the use of the measuring program ImageJ (1.50i, Fiji).

Fabrication of Nanocomposites. The PVA (1 wt \%) was first dissolved in deionized water at $90{ }^{\circ} \mathrm{C}$ followed by the addition of 10 wt \% (based on the mass of PVA) sulfated nanofibers. The mixture was degassed by using an ultrasound bath for $10 \mathrm{~min}$. Solution was then cast on a Petri dish and allowed to dry at room temperature to obtain nanocomposites with a grammage of $60 \mathrm{~g} / \mathrm{m}^{2}$. The pristine PVA film was prepared in a similar manner but without the added nanofibers. 
Mechanical Properties of Self-Standing Films. The tensile tests were performed with a universal material-testing machine (Zwick D0724587, Switzerland) equipped with a $100 \mathrm{~N}$ load cell. The pure PVA and nanocomposite films were cut into $5 \mathrm{~mm}$ wide strips and their thicknesses were measured using a precision thickness gage (Hanatek FT3, UK). Three different locations were measured to calculate the average thickness of each film. For the tensile tests, a 40 $\mathrm{mm}$ gage length was set under a strain rate of $4 \mathrm{~mm} / \mathrm{min}$. Five strips in total were measured for each sample. The tests were conducted in a relative humidity of $50 \%$ at a temperature of $23{ }^{\circ} \mathrm{C}$ using a preload of $\sim 0.1 \mathrm{~N}$. Each sample was conditioned for at least 1 day in this environment before testing. Average thickness of the PVA and SCNFs, SWNFs, and SSDNFs composites were $39.7 \pm 6.5,52.0 \pm$ $1.5,52.0 \pm 1.0$, and $52.7 \pm 1.8 \mu \mathrm{m}$, respectively.

UV-Vis Spectroscopy. The UV-vis spectra of the pure PVA film and the nanocomposites was measured using the UV-2600 spectrophotometer (Shimadzu, Japan). Empty compartment (air) was used as the baseline.

Statistical Analysis. One-way analysis of variance (ANOVA) was conducted using OriginPro 2018 to determine a statistical significant $(p<0.05)$ of the tensile test results.

\section{RESULTS AND DISCUSSION}

Sulfation of GWP and Sawdust. UV-adsorbing WNFs were produced by using unbleached GWP as a commercial lignin-containing raw material. Sawdust, representing the industrial side stream, was studied as the secondary raw material. Because of the large particle size, the sawdust was first ground with an ultracentrifugal mill using a sieve size of 250 $\mu \mathrm{m}$. The chemical composition of both materials was relatively similar: with lignin contents of 27.4 and $28.6 \%$ for GWP and sawdust, respectively (Table 1). Digital images of GWP and original sawdust and ground sawdust are presented in Figure 1.

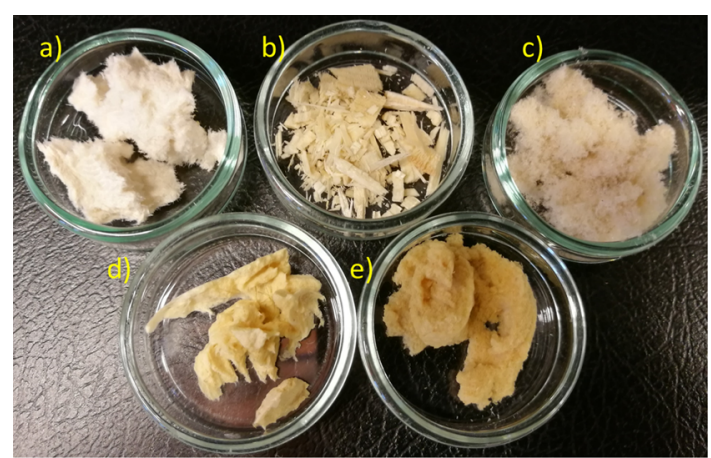

Figure 1. Visual appearance of (a) oven-dried GWP, (b) sawdust, (c) ground sawdust, (d) freeze-dried sulfated GWP, and (e) ground sawdust.

On the basis of the SEM images, the GWP material was heterogeneous and contained large cell wall fragments with diameters of several hundred micrometers along with thinner, fibrillated particles with a diameter of about $10 \mu \mathrm{m}$ (Figure 2). On the other hand, prior to the milling, the sawdust exhibited large hollow particles with dimensions up to $100 \mu \mathrm{m}$. Then, the milling disintegrated the sawdust particles into belt-like fibers with a diameter of approximately $10 \mu \mathrm{m}$ (Figure $2 \mathrm{c}$ ). Compared with the GWP, the ground sawdust particles appeared as larger but a more homogenous material.

Reactive DES was used to pretreat both GWP and ground sawdust before the mechanical liberation of WNFs. Sulfation of the raw materials was conducted by using sulfamic acid and urea DES with a molar ratio of 1:2. The use of sulfamic acid-
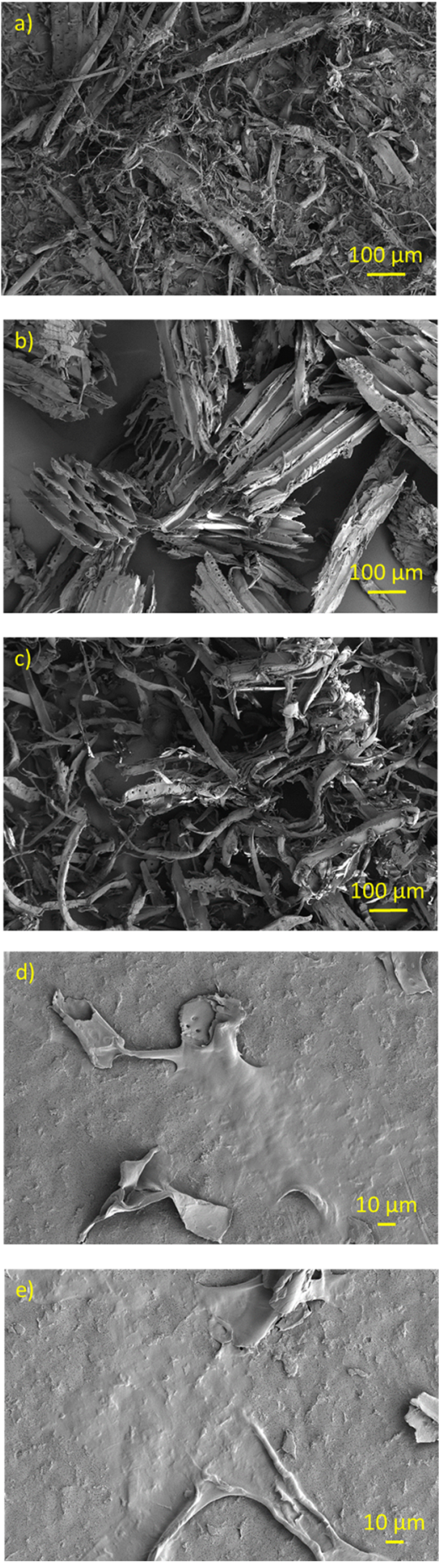

Figure 2. SEM images of raw materials: (a) GWP, (b) sawdust, and (c) sawdust after grinding and raw materials after sulfation: (d) sulfated GWP and (e) sulfated ground sawdust.

based DES led to the attachment of ammonium salt of sulfate ester onto the lignocellulose. In this regard, the sulfation process is believed to occur on the alcohol (hydroxyl) unit of lignocellulose while the aromatic moieties remain unreactive because of the selectivity of sulfamic acid. ${ }^{38}$

The sulfation was performed in an external solvent-free condition, that is, the DES acted as both reaction media and reagent. When both GWP and ground sawdust were subjected to sulfation, the morphology of the particles was significantly altered: it resulted in the disintegration of larger particles and 
in some fibrillation (Figures 1d,e; 2d,e). Although belt-like particles could still be observed, the structure was significantly flattened. The introduction of charged groups into wood particles might have increased the water adsorption and the electrostatic repulsion between the wood components, resulting in swelling and ultimately a spontaneous disintegration of fibers even without mechanical treatment (no mechanical force was applied during the sulfation). The visual appearance of freeze-dried sulfated GWP and ground sawdust were similar (Figure 1) and appeared slightly more yellowish compared with the original wood fibers.

On the basis of the elemental analysis, the amount of sulfate groups was $3.1 \mathrm{mmol} / \mathrm{g}$ for both the GWP and ground sawdust, which was similar to the values obtained from the reference bleached dissolving pulp $(3.0 \mathrm{mmol} / \mathrm{g})$. These results indicate that the presence of lignin and hemicelluloses-at a substantial amount-do not hinder the reactivity of GWP and sawdust with DES compared with the almost pure cellulose fibers (dissolving pulp).

The solvent treatment of cellulose fibers with reactive DES can lead to carbamation of cellulose fibers to some extent. Our results show that the nitrogen content was 4.3 and $4.1 \mathrm{mmol} / \mathrm{g}$ for GWP and ground sawdust, respectively. Here, as the sulfation, which uses sulfamic acid, should lead to a similar nitrogen and sulfur content (i.e., the formation of ammonium salt of sulfate ester), the excess of nitrogen indicates the occurrence of carbamation. In this case-because one nitrogen is introduced by one carbamate group and one nitrogen originates from one ammonium ion of the sulfate group-the carbamate content of sulfated GWP and ground sawdust was around $1 \mathrm{mmol} / \mathrm{g}$.

The yields of the sulfated GWP and ground sawdust were 94 and $92 \%$, respectively, which were notably higher than the bleached cellulose pulp (74\%). The yields of lignin-containing pulps were similar compared with previous results of succinylation of GWP in DES ${ }^{21}$ and slightly higher compared with cationic GWP. ${ }^{39}$ There are several factors that can have an effect on the differences in yields between bleached cellulose pulp and wood fibers. For instance, the chemical treatment during the bleaching significantly reduces the degree of polymerization of cellulose compared with pristine wood. A lower degree of polymerization can lead to a partial dissolution of chemically modified cellulose during the washing step, lowering the yield. In addition, wood fibers also contain other polymers than cellulose (i.e., lignin and hemicellulose), and it can be assumed that all of them react during the sulfation. Therefore, the net charge of cellulose might be lower compared with the dissolving pulp, leading to a lower degree of dissolution during the washing step.

The reactive DES pretreatment was also conducted using sawdust without size reduction via milling. The results showed a sulfate content of $2.1 \mathrm{mmol} / \mathrm{g}$. Nevertheless, sulfated sawdust could not be efficiently disintegrated in water as most of the sample remained as large particles. For this reason, the use of pure sawdust was not researched further. However, it might be possible to do a simultaneous mild mechanical micronization and sulfation as a solution, which will be studied in our future studies.

Chemical Characterization of Sulfated GWP and Sawdust. On the basis of the DRIFT spectra, the chemical characteristics of both GWP and ground sawdust were found to be similar (Figure 3). This was expected as no bleaching was done with these samples. In addition to the characteristic

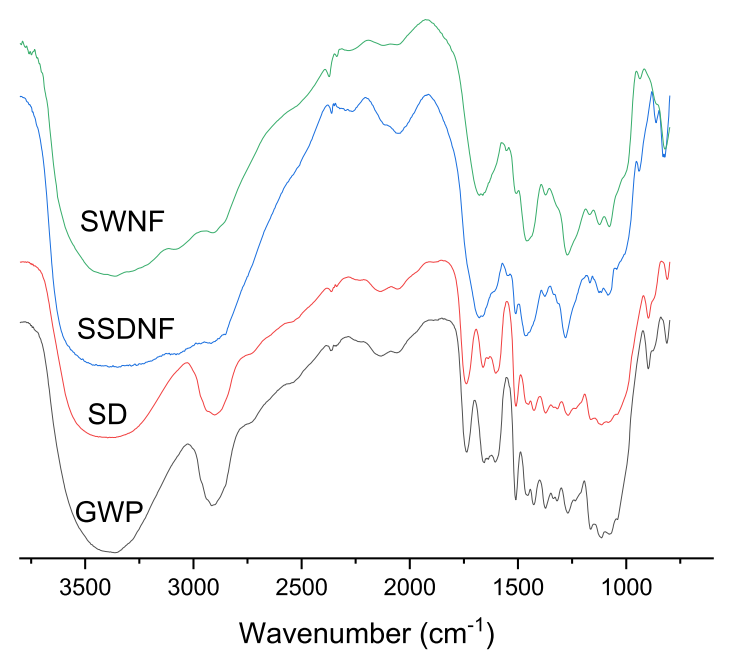

Figure 3. DRIFT spectra of raw materials (GWP and sawdust [SD]) and wood-based nanofibers (SWNF and SSDNF). Nanofibrillation of GWP and sawdust.

cellulose peaks, both samples contained typical peaks related to lignin and hemicelluloses. The sharp peak located at wavenumber $1736 \mathrm{~cm}^{-1}$ is related to the $\mathrm{C}=\mathrm{O}$ stretching of the ester from the acetyl group of hemicellulose and ester linkage of the carboxylic groups in the ferulic and p-coumaric acids of lignin and hemicellulose. Aromatic ring $\mathrm{C}=\mathrm{C}$ stretching peaks of lignin were present at 1605 and 1510 $\mathrm{cm}^{-1}$. After the sulfation, new peaks were seen at 1281 and 821 $\mathrm{cm}^{-1}$, which were related to the asymmetric $\mathrm{S}=\mathrm{O}$ and the symmetric $\mathrm{C}-\mathrm{O}-\mathrm{S}$ vibrations of sulfate groups, respectively. In addition, because of the formation of ammonium salt, the $\mathrm{NH}_{4}^{+}$deformation vibration was observed at $1471 \mathrm{~cm}^{-1}$.

The presence of new peaks indicates that both GWP and ground sawdust were efficiently sulfated by the reactive DES. In addition to the sulfate peaks, a broad peak appeared at a wavenumber of $1678 \mathrm{~cm}^{-1}$ (Figure 3). This peak can be related to the superimposed peak of the carbonyl of lignin and hemicellulose, aromatic ring, bound water, and carbamate group (formed during the sulfation). The carbamate peak typically appeared around $1710 \mathrm{~cm}^{-1}$, and because of the low resolution of DRIFT, it was fused together with the original peaks of unbleached lignocellulose pulps. The broadening of the $\mathrm{OH}-$ and hydrogen bond region around $3300 \mathrm{~cm}^{-1}$ was also observed after the sulfation. Thus, the sulfation most likely alters the hydrogen bonding pattern of wood fibers. In addition, the $\mathrm{N}-\mathrm{H}$ stretching of the ammonium ion and primary amide of carbamate was located at $3300 \mathrm{~cm}^{-1}$, leading into the broadening of the peak observed in this region.

The sulfated pulps (GWP, ground sawdust, and dissolving pulp) were disintegrated into nanosized fibers using a microfluidizer. Here, intensive swelling of chemically modified bleached cellulose pulps can cause problems during the sample feeding into the microfluidizer. Therefore, the sulfated dissolving pulp was diluted to a $0.5 \%$ dispersion before the mechanical disintegration by a microfluidizer. Although both sulfated GWP and ground sawdust exhibited a similar charge density, they were significantly less swollen when placed in water compared with the sulfated dissolving pulp. The presence of hydrophobic lignin might be the main cause for the lower degree of swelling. The disintegration of sulfated wood fibers was performed at a $1 \%$ concentration. We assumed that fibrillation could be performed at an even higher 
concentration level; however, further studies should be conducted to confirm this.

After fibrillation of the sulfated GWP and ground sawdust, a yellow, gel-like suspension was obtained (Figure 4c). In
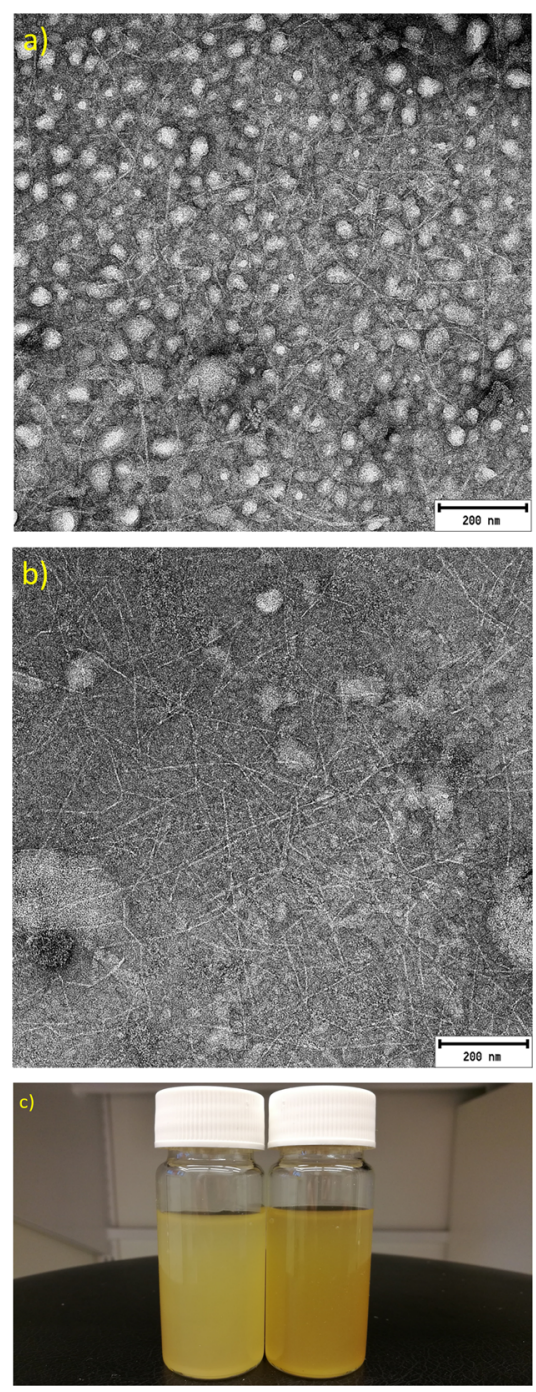

Figure 4. TEM images of (a) SWNFs and (b) SSDNFs and (c) photograph of SWNFs (right bottle) and SSNDFs (left bottle) dispersed directly after fibrillation ( $\sim 0.8 \%$ in water). Ground circles in TEM are due to the sample preparation, most likely due to the air bubbles trapped in the cellulose solution. ${ }^{44}$

general, natural lignin is colorless or slightly yellow; ${ }^{40}$ therefore, sulfation most likely altered the light absorbing properties of lignin, resulting in the formation of a colored dispersion. Regardless of the yellow color, sulfation that uses DES alters the light absorption properties of lignin only mildly compared with industrial processes such as kraft pulping, where an intensively dark-colored pulp is obtained despite the removal of a large quantity of lignin.

The formation of nanosized fibers was confirmed by TEM images as shown in Figure 4 (additional TEM images can be found in the Supporting Information). The sizes of the individual SWNFs and SSDNFs were $2.9 \pm 0.8$ and $3.2 \pm 0.8$ $\mathrm{nm}$, respectively, which is similar to the elemental fibril size and CNFs obtained by chemical modifications such as $(2,2,6,6$ tetramethylpiperidin-1-yl)oxyl-mediated oxidations. ${ }^{41}$ In addi- tion to individual nanofibrils, some larger fiber aggregates were observed (see the Supporting Information), which indicates that the presence of lignin might hinder the complete nanofibrillation of wood fibers. The complete nanofibrillation of bleached cellulose fibers has been observed by using, for example, phosphorylation; ${ }^{42}$ however, it is uncommon to have all the fibers nanofibrillated to nanosized ones. The nanofibrillation efficiency also depends on the severity of mechanical disintegration. ${ }^{23}$ In our study, a relatively mild disintegration was used, and it could be envisioned that remaining fiber aggregates could be disintegrated by using more bypasses through the microfluidizer. Nevertheless, as can be seen in the transmittance of PVA composites, both SWNFs and SSDNFs showed only a minimal effect on the transparency of the nanocomposites in the visible light spectrum (see Nanocomposites section). Thus, further disintegration woodbased nanofibers might even not be necessary for composite applications. In addition to the fiber aggregates, some coarser, uneven nanosized particles were observed as well (see the Supporting Information), which most likely originate from lignin - an observation previously observed. ${ }^{39,43}$

Nanocomposites. PVA is considered a potential material that can be used, for example, as an encapsulation layer for solar cells. ${ }^{45}$ However, PVA has low UV-absorbing properties, which might lead to malfunctioning of the solar cells because of damage induced by UV. For this reason, nanocomposites of PVA containing $10 \mathrm{wt} \%$ of wood-based UV-absorbing additive were produced using the solvent casting technique. After drying, transparent, self-standing films were obtained (Figure 5). Here, our results show that the pristine PVA- and SCNF-

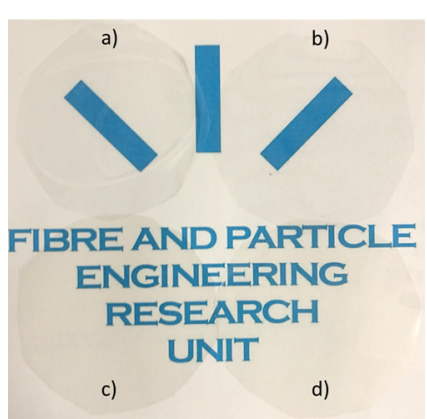

Figure 5. Photograph of (a) pristine PVA, and composites with 10\% of (b) SCNFs, (c) SSDNFs, and (d) SWNFs. The logo is used with permission from University of Oulu. Visible light and UV spectra of nanocomposites.

containing films were colorless, whereas a slightly yellowish tint was observed in films containing SWNFs and SSDNFs. However, the differences between samples were minimal.

Within the visible light spectrum $(380-800 \mathrm{~nm})$, the addition of nanofibers had no notable effect on the total transmittance of PVA (Figure 6). Only at the end of the visible light region, the transmittance of nanocomposite films containing SWNF and SSNDF diminished from $91 \%$ in case of pure PVA to approximately $80 \%$.

The SCNF-containing nanocomposite had a similar transmittance compared with the PVA with the visible light spectrum, and only a small decrease in the transmittance was observed within the UV spectrum (Figure 6). For example, at a wavelength of $280 \mathrm{~nm}$, the pristine PVA had a transmittance of $79 \%$, whereas the transmittance of SCNF-containing nanocomposite was $68 \%$. Both the SWNF- and SSDNF-containing 


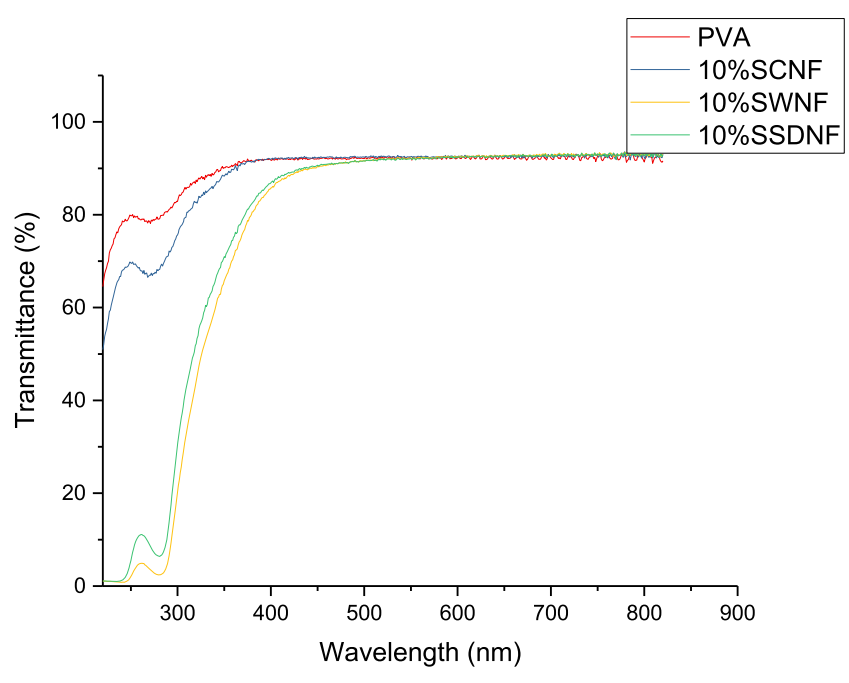

Figure 6. UV-vis spectra of pristine PVA film and nanocomposites.

nanocomposites had significant UV-absorbing capabilities as the transmittance gradually decreased at UVA $(315-400 \mathrm{~nm})$ and UVB (280-315 nm) spectrum, and only minimal transmittances from 2 to $6 \%$ were measured around $280 \mathrm{~nm}$. The significant difference in transmittance between SWNFand SSDNF-containing nanocomposites and both the pristine PVA and SCNF-containing nanocomposite indicates that the lignin functions as a UV-absorbing component. In addition, a small increase in transmittance at the UVC spectrum indicates that the decrease of UV blocking is due to absorption and not because of the UV-radiation blocking caused by larger particles (e.g., via reflection).

Previously, various nanomaterials from different sources have been used as an additive for PVA films to improve UVabsorption properties. For instance, the use of $4 \mathrm{wt} \%$ of lignin nanoparticles was reported to reduce the transmission at the beginning of the UVA region $(400 \mathrm{~nm})$ to around $20 \%$, and a complete UV blocking was observed at the UVB region. ${ }^{46}$ However, a significant reduction in transparency at the visible light region occurred, that is, the transmission at $500 \mathrm{~nm}$ was reduced from the original $90 \%$ to around $50 \%$. This was seen as an intense yellowing of the PVA film. Relatively similar UV absorption properties as wood-based nanofibers were reported for $p$-aminobenzoic acid-containing CNCs (synthesized using a two-step reaction). ${ }^{47}$ A very strong UV-blocking PVA film was previously produced by using a one-pot synthesis of carbon quantum dots, which resulted in a transmittance below $6 \%$ even at a wavelength of $350 \mathrm{~nm} .{ }^{48}$ In this case, a reduced transmittance at the end of the visible light region was observed as well, that is, the transmission at a wavelength of $450 \mathrm{~nm}$ was around $40 \%$. In situ-generated silver nanoparticles efficiently blocked the UV radiation to a near $0 \%$ transmittance-especially after a swift heavy ion irritation-yet a close to zero transmittance was already observed at a wavelength of $500 \mathrm{~nm}^{49}$ As a molecular UV absorbent, natural polyphenols have been used to decrease the UV transmission of PVA films to a near $0 \%$ transmittance; however, the transmittance at $450 \mathrm{~nm}$ also decreased from $90 \%$ to below $30 \%$. $^{50}$ Therefore, both SWNFs and SSDNFs have a potential use as additives for nanocomposites to obtain very high transparency at the visible light spectrum with strong UV-blocking properties, especially at the lower end of the UVA and UVB regions.
Mechanical Properties of Nanocomposites. The present work showed also showed that the nanocomposites were reinforced compared with the pristine PVA film without nanofibers. The modulus of the pristine PVA increased from 1.4 to $1.7 \mathrm{GPa}$ and $1.8 \mathrm{GPa}$ after introducing $10 \mathrm{wt} \%$ of SWNFs and SSDNFs, respectively (Figure 7). In contrast to

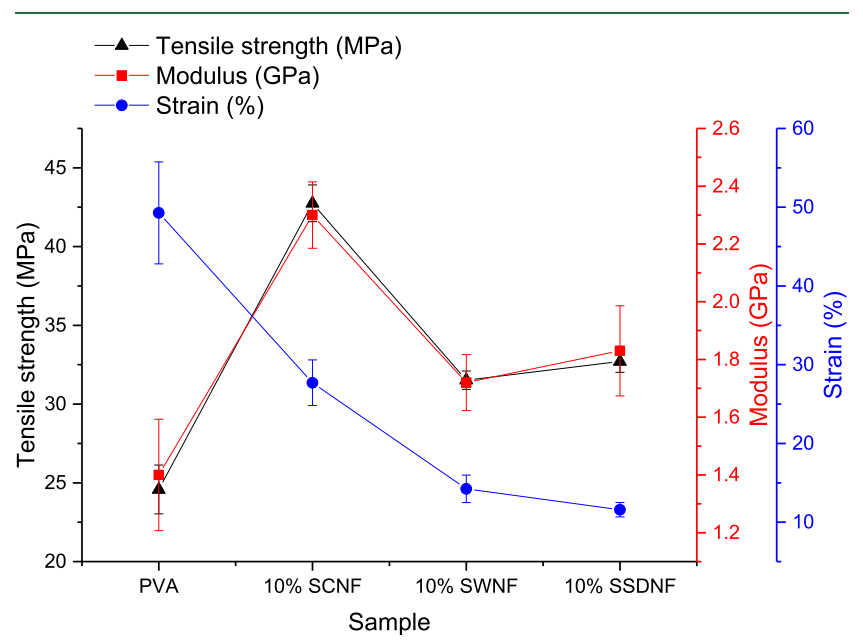

Figure 7. Mechanical properties of pristine PVA and nanocomposite films (error bar represents the standard error).

PVA, the tensile strength of the SWNF- and SSDNFcontaining nanocomposites was 28 and $33 \%$ higher, respectively. On the basis of the ANOVA, there was a significant difference between the tensile strength and modulus of pristine PVA film and SWNF- and SSDNF-containing nanocomposites. However, no significant difference regarding the reinforcement effect between the SWNFs and SSDNFs was observed.

The highest tensile strength and modulus were recorded when 10 wt \% of SCNFs was used in nanocomposites. This indicates that lignin slightly decreased the hydrogen-bonding abilities of SWNFs and SSDNFs, resulting in a somewhat lower mechanical property. Similar to the wood fibers, ${ }^{51}$ lignincontaining nanofibers were previously reported to have a lower tensile strength than nanofibers obtained from bleached cellulose fibers. ${ }^{20}$ In addition, despite having a higher stiffness, nanocomposites containing SCNFs required a higher strain until their breaking point (28\%) compared with SWNF (14\%) and SSDNF (12\%) containing nanocomposites.

Although lignin-containing nanofibers had a lower reinforcement impact compared with the nanofibers produced from bleached cellulose pulp, nanocomposites containing $10 \%$ of SWNFs and SSDNFs had properties similar to other UVblocking films. For example, the addition of $10 \%$-aminobenzoic acid-containing CNCs increased the tensile strength by $33 \%,{ }^{47}$ whereas a 10 and $20 \%$ increase in tensile strength was obtained when using lignin nanoparticles. ${ }^{46}$ On the other hand, a higher improvement on the modulus was achieved when using $10 \%$-aminobenzoic acid-containing CNCs (77\%) compared with the wood-based nanofibers (around 29\%).

\section{CONCLUSIONS}

Both commercial unbleached GWP and sawdust could be used to produce wood-based UV-absorbing additives for nanocomposite applications. Both lignocellulose materials exhibited a similar reactivity compared with the bleached cellulose pulp, 
whereas only minor UV-absorption properties were obtained with SCNFs. Lignin-containing nanofibers, conversely, exhibited strong UV-blocking properties with only a minimal effect on the transmittance at the visible light region of the PVA film. Although SCNFs had a higher reinforcing effect with the PVA matrix, the mechanical properties of the nanocomposites containing wood-based UV-absorbing nanofibers were comparable with many previously studied UV-absorbing nanomaterials. Therefore, both SWNFs and SSDNFs are considered as potential additives for the utilization in applications-such as optical lenses and optoelectronicsthat require high visible light transparency and strong UV blocking.

\section{ASSOCIATED CONTENT}

\section{S Supporting Information}

The Supporting Information is available free of charge on the ACS Publications website at DOI: 10.1021/acs.biomac.9b00427.

TEM images of WNFs at different magnifications (PDF)

\section{AUTHOR INFORMATION}

\section{Corresponding Author}

*E-mail: juho.sirvio@oulu.fi.

\section{ORCID}

Juho Antti Sirviö: 0000-0002-7404-3340

Miikka Visanko: 0000-0001-7480-064X

Notes

The authors declare no competing financial interest.

\section{ACKNOWLEDGMENTS}

We thank Elisa Wirkkala for her assistance with the elemental analysis and Dr. Mika Kaakinen and Sami Saukko for their help with the TEM measurements. We thank Prof. Marko Huttula of the Nano and Molecular Systems Research Unit for the use of the UV-vis spectrometer. The facilities at the Center of Microscopy and Nanotechnology at the University of Oulu were used in this research. The research was conducted as part of the ARVOPURU project funded by the Council of Oulu Region, which was granted by the European Regional Development Fund of the European Union.

\section{REFERENCES}

(1) Klemm, D.; Kramer, F.; Moritz, S.; Lindström, T.; Ankerfors, M.; Gray, D.; Dorris, A. Nanocelluloses: A New Family of Nature-Based Materials. Angew. Chem., Int. Ed. 2011, 50, 5438-5466.

(2) Mohammed, M.; Syeda, J.; Wasan, K.; Wasan, E. An Overview of Chitosan Nanoparticles and Its Application in Non-Parenteral Drug Delivery. Pharmaceutics 2017, 9, 53.

(3) Roopan, S. M. An Overview of Natural Renewable Bio-Polymer Lignin towards Nano and Biotechnological Applications. Int. J. Biol. Macromol. 2017, 103, 508-514.

(4) Le Corre, D.; Bras, J.; Dufresne, A. Starch Nanoparticles: A Review. Biomacromolecules 2010, 11, 1139-1153.

(5) Liu, J.; Cheng, F.; Grénman, H.; Spoljaric, S.; Seppälä, J.; Eriksson, J. E.; Xu, S.; Xu, C. Development of Nanocellulose Scaffolds with Tunable Structures to Support 3D Cell Culture. Carbohydr. Polym. 2016, 148, 259-271.

(6) Lin, N.; Dufresne, A. Nanocellulose in Biomedicine: Current Status and Future Prospect. Eur. Polym. J. 2014, 59, 302-325.

(7) Sirviö, J. A.; Kolehmainen, A.; Visanko, M.; Liimatainen, H.; Niinimäki, J.; Hormi, O. E. O. Strong, Self-Standing Oxygen Barrier
Films from Nanocelluloses Modified with Regioselective Oxidative Treatments. ACS Appl. Mater. Interfaces 2014, 6, 14384-14390.

(8) Aulin, C.; Salazar-Alvarez, G.; Lindström, T. High Strength, Flexible and Transparent Nanofibrillated Cellulose-Nanoclay Biohybrid Films with Tunable Oxygen and Water Vapor Permeability. Nanoscale 2012, 4, 6622.

(9) Hosseini, S. F.; Rezaei, M.; Zandi, M.; Farahmandghavi, F. Fabrication of Bio-Nanocomposite Films Based on Fish Gelatin Reinforced with Chitosan Nanoparticles. Food Hydrocolloids 2015, 44, 172-182.

(10) Oksman, K.; Aitomäki, Y.; Mathew, A. P.; Siqueira, G.; Zhou, Q.; Butylina, S.; Tanpichai, S.; Zhou, X.; Hooshmand, S. Review of the Recent Developments in Cellulose Nanocomposite Processing. Compos. Appl. Sci. Manuf. 2016, 83, 2-18.

(11) Bel Haaj, S.; Thielemans, W.; Magnin, A.; Boufi, S. Starch Nanocrystals and Starch Nanoparticles from Waxy Maize as Nanoreinforcement: A Comparative Study. Carbohydr. Polym. 2016, 143, 310-317.

(12) Ummartyotin, S.; Juntaro, J.; Sain, M.; Manuspiya, H. Development of Transparent Bacterial Cellulose Nanocomposite Film as Substrate for Flexible Organic Light Emitting Diode (OLED) Display. Ind. Crops Prod. 2012, 35, 92-97.

(13) Zhou, Y.; Fuentes-Hernandez, C.; Khan, T. M.; Liu, J.-C.; Hsu, J.; Shim, J. W.; Dindar, A.; Youngblood, J. P.; Moon, R. J.; Kippelen, B. Recyclable Organic Solar Cells on Cellulose Nanocrystal Substrates. Sci. Rep. 2013, 3, 1536.

(14) Missoum, K.; Belgacem, M.; Bras, J. Nanofibrillated Cellulose Surface Modification: A Review. Materials 2013, 6, 1745-1766.

(15) Trache, D.; Hussin, M. H.; Haafiz, M. K. M.; Thakur, V. K. Recent Progress in Cellulose Nanocrystals: Sources and Production. Nanoscale 2017, 9, 1763-1786.

(16) Beaumont, M.; Nypelö, T.; König, J.; Zirbs, R.; Opietnik, M.; Potthast, A.; Rosenau, T. Synthesis of Redispersible Spherical Cellulose II Nanoparticles Decorated with Carboxylate Groups. Green Chem. 2016, 18, 1465-1468.

(17) Sirviö, J. A. Fabrication of Regenerated Cellulose Nanoparticles by Mechanical Disintegration of Cellulose after Dissolution and Regeneration from a Deep Eutectic Solvent. J. Mater. Chem. A 2019, 7, 755-763.

(18) Spence, K. L.; Venditti, R. A.; Habibi, Y.; Rojas, O. J.; Pawlak, J. J. The Effect of Chemical Composition on Microfibrillar Cellulose Films from Wood Pulps: Mechanical Processing and Physical Properties. Bioresour. Technol. 2010, 101, 5961-5968.

(19) Solala, I.; Volperts, A.; Andersone, A.; Dizhbite, T.; MironovaUlmane, N.; Vehniäinen, A.; Pere, J.; Vuorinen, T. Mechanoradical Formation and Its Effects on Birch Kraft Pulp during the Preparation of Nanofibrillated Cellulose with Masuko Refining. Holzforschung 2012, 66, 477-483.

(20) Visanko, M.; Sirviö, J. A.; Piltonen, P.; Sliz, R.; Liimatainen, H.; Illikainen, M. Mechanical Fabrication of High-Strength and Redispersible Wood Nanofibers from Unbleached Groundwood Pulp. Cellulose 2017, 24, 4173-4187.

(21) Sirviö, J. A.; Visanko, M. Anionic Wood Nanofibers Produced from Unbleached Mechanical Pulp by Highly Efficient Chemical Modification. J. Mater. Chem. A 2017, 5, 21828-21835.

(22) Herrera, M.; Thitiwutthisakul, K.; Yang, X.; Rujitanaroj, P.; Rojas, R.; Berglund, L. Preparation and Evaluation of High-Lignin Content Cellulose Nanofibrils from Eucalyptus Pulp. Cellulose 2018, $1-13,3121-3133$

(23) Hassan, M.; Berglund, L.; Hassan, E.; Abou-Zeid, R.; Oksman, K. Effect of Xylanase Pretreatment of Rice Straw Unbleached Soda and Neutral Sulfite Pulps on Isolation of Nanofibers and Their Properties. Cellulose 2018, 1-15, 2939-2953.

(24) Bian, H.; Gao, Y.; Wang, R.; Liu, Z.; Wu, W.; Dai, H. Contribution of Lignin to the Surface Structure and Physical Performance of Cellulose Nanofibrils Film. Cellulose 2018, 1-10, $1309-1318$ 
(25) Suopajärvi, T.; Liimatainen, H.; Karjalainen, M.; Upola, H.; Niinimäki, J. Lead Adsorption with Sulfonated Wheat Pulp Nanocelluloses. J. Water Process Eng. 2015, 5, 136-142.

(26) Guo, X.; Zhang, S.; Shan, X.-q. Adsorption of Metal Ions on Lignin. J. Hazard. Mater. 2008, 151, 134-142.

(27) Pouteau, C.; Dole, P.; Cathala, B.; Averous, L.; Boquillon, N. Antioxidant Properties of Lignin in Polypropylene. Polym. Degrad. Stab. 2003, 81, 9-18.

(28) Li, Z.; Ge, Y. Antioxidant Activities of Lignin Extracted from Sugarcane Bagasse via Different Chemical Procedures. Int. J. Biol. Macromol. 2012, 51, 1116-1120.

(29) Heiskanen, I.; Lyytikäinen, K.; Saukkonen, E.; Backfolk, K. A Uv Blocking Film and Composition Comprising Microfibrillated Cellulose, a Method for Producing Said Film and Use of the Composition. WO2017/199157, Nov 24, 2017.

(30) Sadeghifar, H.; Venditti, R.; Jur, J.; Gorga, R. E.; Pawlak, J. J. Cellulose-Lignin Biodegradable and Flexible UV Protection Film. ACS Sustain. Chem. Eng. 2017, 5, 625-631.

(31) Lin, K.-K.; Lin, Y.-C.; Lee, J.-S.; Chao, A.-N.; Chen, H. S.-L. Spectral Transmission Characteristics of Spectacle Contact, and Intraocular Lenses. Ann. Ophthalmol. 2002, 34, 206-215.

(32) Cheng, P.; Zhan, X. Stability of Organic Solar Cells: Challenges and Strategies. Chem. Soc. Rev. 2016, 45, 2544-2582.

(33) Tomović, A. Ž.; Jovanović, V. P.; Durišić, I.; Cerovski, V. Z.; Nastasijević, B.; Veličković, S. R.; Radulović, K.; Žikić, R. Fast Photoluminescence Quenching in Thin Films of 4,4'-Bis(2,2Diphenylvinyl)-1,1'-Biphenyl Exposed to Air. J. Lumin. 2015, 167, 204-210.

(34) Sirvio, J.; Hyvakko, U.; Liimatainen, H.; Niinimaki, J.; Hormi, O. Periodate Oxidation of Cellulose at Elevated Temperatures Using Metal Salts as Cellulose Activators. Carbohydr. Polym. 2011, 83, $1293-1297$.

(35) Sirviö, J. A.; Ukkola, J.; Liimatainen, H. Direct Sulfation of Cellulose Fibers Using a Reactive Deep Eutectic Solvent to Produce Highly Charged Cellulose Nanofibers. Cellulose 2019, 26, 2303-2316.

(36) Levdansky, V. A.; Kondracenko, A. S.; Levdansky, A. V.; Kuznetsov, B. N.; Djakovitch, L.; Pinel, C.; et al. Sulfation of Microcrystalline Cellulose with Sulfamic Acid in N,N-Dimethylformamide and Diglyme. J. Sib. Fed. Univ., Chem. 2014, 2, 162-169.

(37) Tingaut, P.; Hauert, R.; Zimmermann, T. Highly Efficient and Straightforward Functionalization of Cellulose Films with Thiol-Ene Click Chemistry. J. Mater. Chem. 2011, 21, 16066-16076.

(38) Benson, G. A.; Spillane, W. J. Sulfamic Acid and Its NSubstituted Derivatives. Chem. Rev. 1980, 80, 151-186.

(39) Sirviö, J. A. Cationization of Lignocellulosic Fibers with Betaine in Deep Eutectic Solvent: Facile Route to Charge Stabilized Cellulose and Wood Nanofibers. Carbohydr. Polym. 2018, 198, 34-40.

(40) Zhang, H.; Bai, Y.; Zhou, W.; Chen, F. Color Reduction of Sulfonated Eucalyptus Kraft Lignin. Int. J. Biol. Macromol. 2017, 97, 201-208.

(41) Isogai, A.; Saito, T.; Fukuzumi, H. TEMPO-Oxidized Cellulose Nanofibers. Nanoscale 2011, 3, 71-85.

(42) Noguchi, Y.; Homma, I.; Matsubara, Y. Complete Nanofibrillation of Cellulose Prepared by Phosphorylation. Cellulose 2017, 24, 1295-1305.

(43) Bian, H.; Chen, L.; Dai, H.; Zhu, J. Y. Integrated Production of Lignin Containing Cellulose Nanocrystals (LCNC) and Nanofibrils (LCNF) Using an Easily Recyclable Di-Carboxylic Acid. Carbohydr. Polym. 2017, 167, 167-176.

(44) Sirviö, J. A.; Visanko, M.; Liimatainen, H. Acidic Deep Eutectic Solvents As Hydrolytic Media for Cellulose Nanocrystal Production. Biomacromolecules 2016, 17, 3025-3032.

(45) Gaume, J.; Wong-Wah-Chung, P.; Rivaton, A.; Thérias, S.; Gardette, J.-L. Photochemical Behavior of PVA as an Oxygen-Barrier Polymer for Solar Cell Encapsulation. RSC Adv. 2011, 1, 1471-1481.

(46) Tian, D.; Hu, J.; Bao, J.; Chandra, R. P.; Saddler, J. N.; Lu, C. Lignin Valorization: Lignin Nanoparticles as High-Value Bio-Additive for Multifunctional Nanocomposites. Biotechnol. Biofuels 2017, 10, 192.
(47) Sirviö, J. A.; Visanko, M.; Heiskanen, J. P.; Liimatainen, H. UVAbsorbing Cellulose Nanocrystals as Functional Reinforcing Fillers in Polymer Nanocomposite Films. J. Mater. Chem. A 2016, 4, 63686375.

(48) Hess, S. C.; Fukazawa, F. A.; Fukazawa, H.; Schneider, E. M.; Balgis, R.; Ogi, T.; Okuyama, K.; Stark, W. J. Direct Synthesis of Carbon Quantum Dots in Aqueous Polymer Solution: One-Pot Reaction and Preparation of Transparent UV-Blocking Films. J. Mater. Chem. A 2017, 5, 5187-5194.

(49) Chahal, R. P.; Mahendia, S.; Tomar, A. K.; Kumar, S. SHI Irradiated PVA/Ag Nanocomposites and Possibility of UV Blocking. Opt. Mater. 2016, 52, 237-241.

(50) Xu, X.-J.; Huang, S.-M.; Zhang, L.-H. Biodegradability, Antibacterial Properties, and Ultraviolet Protection of Polyvinyl Alcohol-Natural Polyphenol Blends. Polym. Compos. 2009, 30, 16111617.

(51) Zhang, S.-Y.; Fei, B.-H.; Yu, Y.; Cheng, H.-T.; Wang, C.-G. Effect of the Amount of Lignin on Tensile Properties of Single Wood Fibers. For. Sci. Pract. 2013, 15, 56-60. 